\title{
Diagnostic tests for Cushing's syndrome differ from published guidelines: data from ERCUSYN
}

\author{
Elena Valassi ${ }^{1}$, Holger Franz ${ }^{2}$, Thierry Brue ${ }^{3}$, Richard A Feelders $^{4}$, Romana Netea-Maier ${ }^{5}$, \\ Stylianos Tsagarakis ${ }^{6}$, Susan M Webb ${ }^{1}$, Maria Yaneva7, Martin Reincke ${ }^{8}$, Michael Droste ${ }^{9}$, \\ Irina Komerdus ${ }^{10}$, Dominique Maiter ${ }^{11}$, Darko Kastelan ${ }^{12}$, Philippe Chanson ${ }^{13,14,15}$, Marija Pfeifer ${ }^{16}$, \\ Christian J Strasburger ${ }^{17}$, Miklós Tóth ${ }^{18}$, Olivier Chabre ${ }^{19}$, Antoine Tabarin ${ }^{20}$, Michal Krsek ${ }^{21}$, \\ Carmen Fajardo ${ }^{22}$, Marek Bolanowski ${ }^{23}$, Alicia Santos ${ }^{1}$, John A H Wass ${ }^{24}$ and Peter J Trainer ${ }^{25}$, for \\ the ERCUSYN Study Group ${ }^{+}$
}

${ }^{1}$ IIB-Sant Pau and Department of Endocrinology/Medicine, Hospital Sant Pau, UAB, and Centro de Investigación Biomédica en Red de Enfermedades Raras (CIBER-ER, Unidad 747), ISCIII, Barcelona, Spain, ${ }^{2}$ Lohmann \& Birkner Health Care Consulting GmbH, Berlin, Germany, ${ }^{3}$ Aix-Marseille Université, CNRS, CRN2M UMR 7286, and APHM, Hôpital Conception, Marseille, France, ${ }^{4}$ Erasmus University Medical Centre, Rotterdam, The Netherlands, ${ }^{5}$ Radboud University Medical Centre, Nijmegen, The Netherlands, ${ }^{6} \mathrm{Athens}$ Polyclinic General Hospital, Evangelismos Hospital, Athens, Greece, ${ }^{7}$ Medical University of Sofia, Sofia, Bulgary, ${ }^{8}$ Medizinische Klinik und Poliklinik IV, Campus Innestadt, Klinikum der Universität München, München, Germany, ${ }^{9}$ Praxis für Endokrinologie Droste, Oldenburg, Germany, ${ }^{10}$ Moscow Regional Research Clinical Institute, Moscow, Russia, ${ }^{11}$ UCL Cliniques Universitaires St Luc, Brussels, Belgium, ${ }^{12}$ Department of Endocrinology, University Hospital Zagreb, School of Medicine University of Zagreb, Zagreb, Croatia, ${ }^{13}$ Univ Paris-Sud, Université Paris-Saclay UMR-S1185, Le Kremlin Bicêtre, Paris, France, ${ }^{14}$ Assistance Publique-Hôpitaux de Paris, Hôpital de Bicêtre, Service de Endocrinologie et des Maladies de la Reproduction, Le Kremlin Bicêtre, Paris, France, ${ }^{15}$ Institut National de la Santé et de la Recherche Médicale U1185, Le Kremlin Bicêtre, Paris, France, ${ }^{16}$ Department of Endocrinology, University Medical Centre Ljubljana, Ljubljana, Slovenia, ${ }^{17}$ Division of Clinical Endocrinology, Department of Medicine CCM, Charité-Universitätsmedizin, Berlin, Germany, ${ }^{18}$ 2nd Department of Medicine, Semmelweis University, Budapest, Hungary, ${ }^{19}$ Service d'Endocrinologie-Diabétologie-Nutrition, Grenoble Cedex, France, ${ }^{20}$ Centre Hospitalier Universitaire de Bordeaux, Bordeaux, France, ${ }^{21}$ 2nd Department of Internal Medicine, 3rd Faculty of Medicine and University Hospital Kralovske Vinohrady, Prague, Czech Republic, ${ }^{22}$ Department of Endocrinology, Hospital Universitario de la Ribera, Alzira, Spain, ${ }^{23}$ Department of Endocrinology, Diabetology and Isotope Therapy, Wroclaw Medical University, Wroclaw, Poland, ${ }^{24}$ Oxford Radcliffe Hospitals NHS Trust, Oxford, UK, and ${ }^{25}$ Department of Endocrinology, Christie Hospital, Manchester, UK ${ }^{\dagger}$ (Details of the ERCUSYN Study Group is presented in Acknowledgements section)

Correspondence should be addressed to S M Webb Email swebb@santpau.cat

\begin{abstract}
Objective: To evaluate which tests are performed to diagnose hypercortisolism in patients included in the European Registry on Cushing's syndrome (ERCUSYN), and to examine if their use differs from the current guidelines.

Patients and methods: We analyzed data on the diagnostic tests performed in 1341 patients with Cushing's syndrome (CS) who have been entered into the ERCUSYN database between January 1, 2000 and January 31, 2016 from 57 centers in 26 European countries. Sixty-seven percent had pituitary-dependent CS (PIT-CS), 24\% had adrenaldependent CS (ADR-CS), $6 \%$ had CS from an ectopic source (ECT-CS) and 3\% were classified as having CS from other causes (OTH-CS).

Results: Of the first-line tests, urinary free cortisol (UFC) test was performed in $78 \%$ of patients, overnight $1 \mathrm{mg}$ dexamethasone suppression test (DST) in $60 \%$ and late-night salivary cortisol (LSaC) in $25 \%$. Use of LSaC increased in the last five years as compared with previous years $(P<0.01)$. Use of HDDST was slightly more frequent in the last 5 years as compared with previous years $(P<0.05)$. Of the additional tests, late-night serum cortisol (LSeC) was measured in $62 \%$ and $48-\mathrm{h} 2 \mathrm{mg} /$ day low-dose dexamethasone suppression test (LDDST) in $33 \%$ of cases. ACTH was performed in $78 \%$ of patients. LSeC and overnight $1 \mathrm{mg}$ DST supported the diagnosis of both PIT-CS and ADR-CS more frequently than UFC $(P<0.05)$.
\end{abstract}

() 2017 European Society of Endocrinology Printed in Great Britain
Published by Bioscientifica Ltd. 
Conclusions: Use of diagnostic tests for CS varies across Europe and partly differs from the currently available guidelines. It would seem pertinent that a European consensus be established to determine the best diagnostic approach to CS, taking into account specific inter-country differences with regard to the availability of diagnostic tools.

\section{Introduction}

Diagnosis of Cushing's syndrome (CS) is a major clinical issue, due to the rarity of this condition and its variable clinical presentation (1). Several biochemical tests have been proposed for the evaluation of suspected hypercortisolism $(2,3,4,5,6,7,8,9,10,11,12)$, but their diagnostic accuracy depends upon etiology of CS, patient's comorbidities, robustness of the assays, concomitant medications and the setting of investigations $(13,14,15$, $16,17,18,19,20,21,22,23$ ).

According to the most recent clinical practice guidelines (24) developed under the auspice of the Endocrine Society in 2008, initial work-up in patients suspected of having CS should rely on one test with high sensitivity, such as urinary free cortisol (UFC); late-night salivary cortisol (LSaC); overnight $1 \mathrm{mg}$ dexamethasone suppression test (DST) or, in some selected subjects, 48-h $2 \mathrm{mg}$ /day low-dose dexamethasone suppression test (LDDST). In case of abnormal results, patients should undergo a second test, either one of the above or, in some cases, a late-night serum cortisol (LSeC) or a dexamethasone-suppressed CRH stimulation (Dex-CRH) test (24). However it is still not known to which extent these recommendations are followed and/or shared on a large-scale by specialists dealing with patients suspected to have endogenous hypercortisolism.

The European Registry on Cushing's syndrome (ERCUSYN) is the largest prospective database existing to date which collects information on diagnosis, management and long-term follow-up in CS (25). Because ERCUSYN includes data from 57 centers in 26 European countries, it reflects the 'real-life' clinical practice and shows which tests are more frequently used to identify CS throughout Europe.

Thus, the aim of this study was to evaluate how CS is diagnosed in Europe and examine if their use differs from the current guidelines. In addition, we have compared the diagnostic strategies used to identify the different etiologic groups of CS.

\section{Patients and methods}

\section{Description of the database}

ERCUSYN is a web-based, multicenter, observational study that enrolled 1386 patients from 57 centers in 26 European countries diagnosed between January 1, 2000 and January 31, 2016. Forty-five patients were excluded due to lack of a definitive diagnosis, so 1341 patients were finally analyzed. One-thousand and fourteen patients were prospectively included since October 1, 2008 (when the database was opened), and 327 patients diagnosed since January 1, 2000 were retrospectively entered with yearly updates. Patients were classified into four major etiologic groups: pituitary-dependent CS (PIT-CS), adrenal-dependent CS (ADR-CS; adrenal adenoma), CS from an ectopic source (ECT-CS) and CS from other etiologies (OTH-CS) (Fig. 1).

Etiologic classification was based on histologic documentation of ACTH-secreting or adrenal tumor. In case histological reports were not available, biochemical and clinical resolution of hypercortisolism after surgical resection were used as a diagnostic confirmation. In patients with ECT-CS or OTH-CS who were not operated on, diagnosis was based on biochemical test results and/or imaging, as confirmed by the managing physician.

A detailed description of the database layout has been previously reported (25). This study was aimed at analyzing and evaluating data entered in the 'diagnosis' section.

The 'diagnosis' section contains information at diagnosis: baseline demographic and anthropometric characteristics, etiology of CS and diagnosis date, delay between onset of symptoms and final diagnosis of CS, other specialists consulted for Cushing's symptoms before correct diagnosis, clinical features, comorbidities, bone status and two questionnaires on quality of life (CushingQoL and EuroQoL-5D). It also contains a subsection comprising the following diagnostic tests: urinary free cortisol (UFC), morning serum cortisol (MSeC), late-night salivary cortisol 


\section{ERCUSYN patients included}

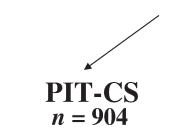$$
\text { AD }
$$

'supporting' or 'not supporting' the pituitary origin of CS.

If a specific item was not available, participants were asked to select 'not answered' (i.e. when information was missing) or 'not known' (when a test or clinical evaluation had been performed but results were not available for any reason).

The ERCUSYN study was approved by the ethics committee of the Hospital de Sant Pau, Barcelona, Spain, which is the coordinating center. In addition, local ethics committee approval was obtained for each participating institution and all patients gave their written informed consent, depending on national legal requirements.

All the data reported into the system were carefully

\section{Figure 1}

Total number of ERCUSYN patients analyzed, divided by etiologic group. CS, Cushing's syndrome; PIT-CS, pituitarydependent Cushing's syndrome; ADR-CS, adrenal-dependent Cushing's syndrome; ECT-CS, ectopic Cushing's syndrome; OTH-CS, Cushing's syndrome from other causes. ADR-CS includes 6 adrenal hyperplasia, 6 primary pigmented nodular adrenocortical disease (PPNAD) and 3 ACTH-independent macronodular adrenocortical hyperplasia (AIMAH). n/a, not available.

(LSaC), late-night serum cortisol (LSeC), overnight $1 \mathrm{mg}$ dexamethasone suppression test (overnight $1 \mathrm{mg}$ DST), and 48-h $2 \mathrm{mg} /$ day low-dose dexamethasone suppression test (LDDST). The following tests were also included for the differential diagnosis: ACTH, 48-h $8 \mathrm{mg} /$ day highdose dexamethasone suppression test (HDDST) (postdexamethasone concentrations of ACTH, serum cortisol, UFC), LSaC, and CRH test (post-CRH concentrations of ACTH and serum cortisol). A blank space was also available for any additional test. For each test, hormone concentrations, units and diagnostic interpretation ('supporting' or 'not supporting' the diagnosis) were required. Space for three UFC values was provided. The average of all the values entered was calculated before analyzing the data.

The 'imaging' subsection contains information on pituitary MRI or CT ('microadenoma,' 'extrasellar macroadenoma,' 'intrasellar macroadenoma' and 'not seen') and adrenal MRI, CT, ultrasounds or endoscopic ultrasounds ('adenoma' in 'left adrenal' and/or 'right adrenal'). In case of ectopic ACTH secretion, available results of MRI, PET, Octreo-Scan or other imaging techniques, and information on the 'region of presumed source of ectopic' as well as 'histology confirmation' were collected. The results of bilateral inferior petrosal sinus sampling (IPSS) were qualitatively described as monitored for inconsistencies, queried when necessary and validated before statistical analysis.

\section{Statistical analysis}

SPSS for Windows version 22.0 (SPSS) was used to perform data analysis. Data on age, BMI and delay to diagnosis are expressed as median (interquartile range (IQR)).

Statistical comparisons were carried out using MannWhitney's $U$ test for quantitative variables and the $\chi^{2}$ for categorical variables. Comparisons between the etiologic groups were performed by ANOVA followed by Bonferroni test as a post hoc test or a Kruskal-Wallis $H$ test, depending on the data distribution. To evaluate if the use of a given diagnostic test changed over time, an arbitrary cut-point of 5 years was established and the number of tests in the last 5 years of the ERCUSYN (2010-2015) was compared with the number of tests prior to 2010 (2000-2009). The diagnostic performance of tests within each etiologic group was performed using the McNemar test. The diagnostic performance of tests was compared between the etiologic groups calculating the likelihood ratio from contingency tables; significance was identified using the adjusted residual. Statistical significance was defined as a two-tailed $P$ value $\leq 0.05$.

\section{Results}

\section{General characteristics}

Of the 1341 patients, 904 (67\%) had PIT-CS, 335 (25\%) had ADR-CS and 80 (6\%) had ECT-CS. Twenty-two (2\%) were classified as having OTH-CS (Fig. 1).

The characteristics of the population and the putative delay to diagnosis for each etiologic group are shown in Table 1. 
Table 1 General characteristics of 1341 CS patients included in the ERCUSYN.

\begin{tabular}{|c|c|c|c|c|c|}
\hline Characteristics & PIT-CS & ADR-CS & ECT-CS & OTH-CS & Total \\
\hline$n(\%)$ & 904 (67) & $335(25)$ & $80(6)$ & $22(2)$ & 1341 \\
\hline Gender (F/M) & $721 / 183$ & $283 / 52$ & $33 / 80$ & $18 / 4$ & $1055 / 286$ \\
\hline$\%$ Males & 20 & 16 & $59^{\S}$ & 18 & 21 \\
\hline $\begin{array}{l}\text { Age at } \\
\text { diagnosis } \\
\text { (year) }^{\dagger}\end{array}$ & $43(20)$ * & $46(22)$ & $49(24)$ & $45(30)$ & $44(21)$ \\
\hline BMI $\left(\mathrm{kg} / \mathrm{m}^{2}\right)^{\dagger}$ & $28(9)$ & $29(8)$ & $26(8) * *$ & 29 (10) & $28(9)$ \\
\hline $\begin{array}{l}\text { Delay to } \\
\text { diagnosis } \\
\text { (year) }^{\dagger}\end{array}$ & $2(3)$ & $2(2)$ & $1(1.5)$ & $1(3)$ & $2(3)$ \\
\hline
\end{tabular}

${ }^{\dagger}$ Values are presented as median (IQR); *PIT-CS patients were younger at diagnosis vs both ADR-CS and ECT-CS $(P<0.01)$; **ECT-CS had lower BMI vs the other groups $(P<0.01)$; 5 proportion of males was higher in ECT-CS vs PIT-CS and ADR-CS $(P<0.05)$.

ADR-CS, adrenal-dependent Cushing's syndrome; ECT-CS, ectopic Cushing's syndrome; OTH-CS, Cushing's syndrome from other causes and IQR, interquartile range; PIT-CS, pituitary-dependent Cushing's syndrome.

\section{Specific country characteristics}

A total of 26 European countries participated in the ERCUSYN (Supplementary Table 1, see section on supplementary data given at the end of this article). Four countries provided more than half of the patients of the database (France $(n=247 ; 18 \%)$, the Netherlands $(n=198$; $15 \%)$, Germany $(13 \% ; n=174)$ and Bulgaria $(n=88 ; 7 \%))$.

\section{Biochemical assessment}

Among the first-line diagnostic tests, UFC was performed across Europe in $78 \%$ of patients, overnight $1 \mathrm{mg}$ DST in $60 \%$ and $\mathrm{LSaC}$ in $20 \%$. The LSeC measurement was used in the diagnostic work-up of $62 \%$ of patients. LDDST was used in 33\% of cases (Table 2 and Supplementary Table 1). ACTH assessment was performed in $78 \%$ of patients. MSeC was reported in $82 \%$ of patients.

When countries contributing with at least 20 patients were considered, the use of UFC ranged from 13 to $99 \%$, LSeC from 15 to $96 \%$, overnight $1 \mathrm{mg}$ DST from to 12 to $90 \%$ and LSaC from to 0 to $46 \%$. The use of LDDST ranged from 3 to $81 \%$ and that of ACTH from 43 to $98 \%$. Dex-CRH test was used in one PIT-CS patient only. Both LSaC and LSeC were more frequently measured in those centers entering more than 20 patients (306/335 (91\%) for LSaC and $738 / 829$ (89\%) for LSeC) as compared with those entering less than 20 patients $(29 / 335$ (9\%) for LSaC and $91 / 829$ (11\%) for LSeC) in the ERCUSYN $(P<0.01$ for both comparisons).
Table 2 Testing recommended or not recommended by the Endocrine Society Guidelines (24), which has been performed in the ERCUSYN centers to diagnose Cushing's syndrome. All values are expressed as a percentage of the number of each performed test with the available information.

\begin{tabular}{|c|c|}
\hline & $\begin{array}{l}\text { Tests performed in the } \\
\text { ERCUSYN centers (\%) }\end{array}$ \\
\hline \multicolumn{2}{|l|}{$\begin{array}{l}\text { Tests recommended by the } \\
\text { Endocrine Society Clinical Practice } \\
\text { Guidelines }\end{array}$} \\
\hline UFC & 78 \\
\hline Overnight $1 \mathrm{mg}$ DST & 60 \\
\hline $\mathrm{LSaC}$ & 25 \\
\hline LDDST (in certain populations) & 33 \\
\hline Dex-CRH (in certain populations) & 0.07 \\
\hline LSeC (in certain populations) & 62 \\
\hline \multicolumn{2}{|l|}{ Not recommended tests } \\
\hline $\mathrm{MSeC}$ & $\mathrm{n} / \mathrm{a}^{*}$ \\
\hline ACTH & 78 \\
\hline HDDST (serum cortisol) & 30 \\
\hline CRH test (cortisol, peak) & 28 \\
\hline
\end{tabular}

*Because MSeC may have been performed in different contexts (e.g. basal assessment of cortisol within LDDST or HDDST testing, cortisol diurnal rhythm, independent measure), we have omitted this information.

DST, dexamethasone suppression test; Dex-CRH, dexamethasonesuppressed CRH stimulation test; HDDST, high-dose dexamethasone suppression test; LDDST, low-dose dexamethasone suppression tests; $\mathrm{LSaC}$, late-night salivary cortisol; $\mathrm{LSeC}$, late-night serum cortisol; $\mathrm{MSeC}$, morning serum cortisol; n/a, not available; UFC, urinary free cortisol.

\section{Use of tests over the time}

The use of LSaC measurement was more frequent in those patients seen in the last 5 years (2010-2015) (194/629 (31\%)) as compared with those seen in the previous years $(2000-2010)(141 / 712(20 \%))(P<0.01)$ (Fig. 2A). The use of HDDST was slightly more frequent in the last 5 years as compared with the previous years (192/399 (48\%) vs 207/399 (52\%); $P=0.018$ ) (Fig. 2B). The use of CRH test was less frequent in the last 5 years as compared with the previous years (155/372 (42\%) vs $217 / 372$ (58\%); $P=0.017$ ) (Fig. 2C).

\section{Comparison of tests supporting the diagnosis of CS within each etiologic group}

The diagnostic performance of tests within each etiologic group is shown in Fig. 3.

In PIT-CS, LSeC (538/544 (99\%)) supported the diagnosis more frequently than both UFC (670/705 (95\%)) and LSaC (183/205 (89\%)); $P<0.01$ for both comparisons. Overnight $1 \mathrm{mg}$ DST (520/531 (98\%)) supported the 


\section{Biochemical tests used for the differential diagnosis of CS}
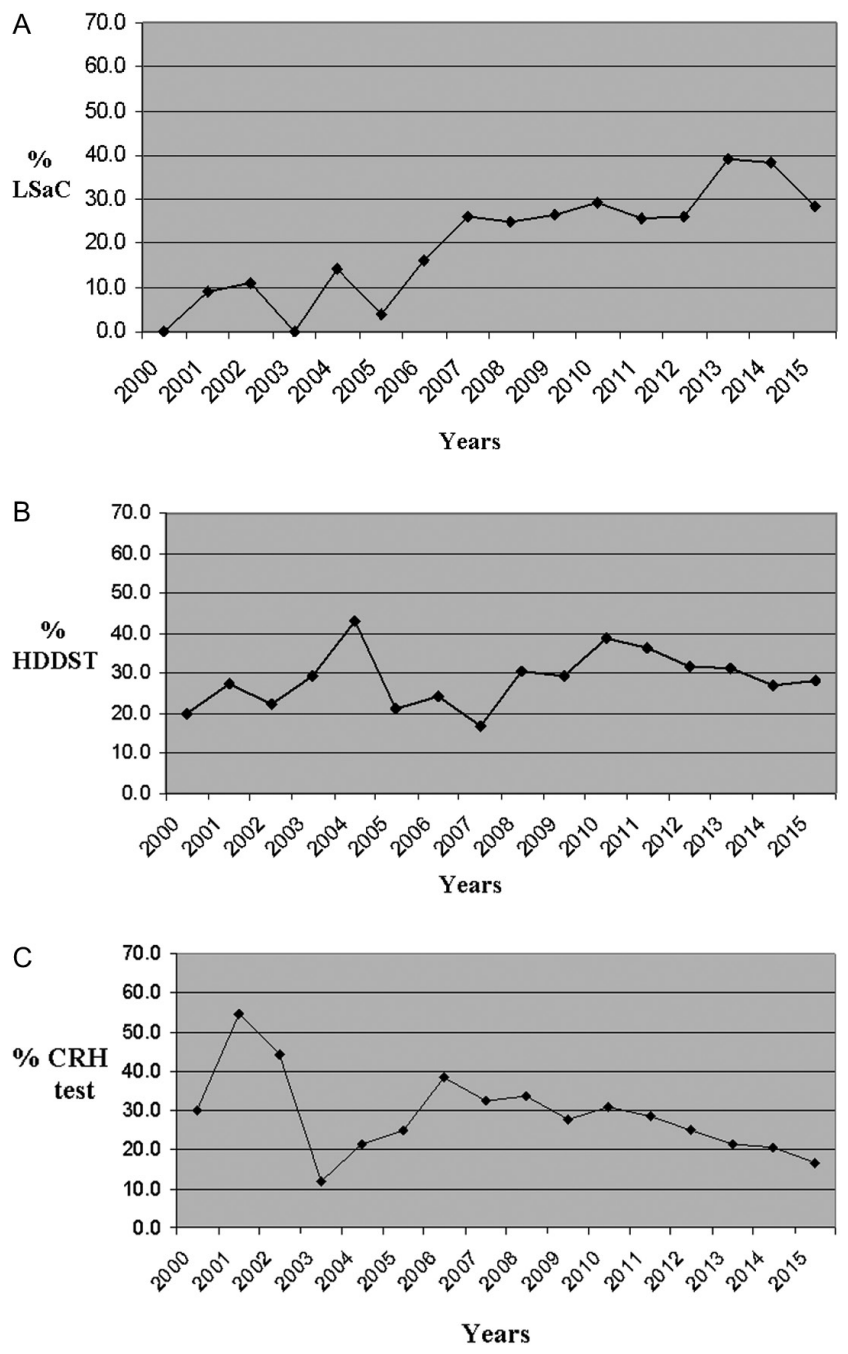

\section{Figure 2}

Trend of the use of diagnostic tests in the ERCUSYN patients over the period 2000-2015. (A) Use of midnight salivary cortisol (LSaC) measurement in ERCUSYN patients has increased over time $(P<0.01)$. (B) Use of high-dose dexamethasone suppression test (HDDST) in ERCUSYN patients has increased over time $(P<0.05)$. (C) Use of CRH test (cortisol) in ERCUSYN patients has decreased over time $(P<0.05)$.

diagnosis more frequently than both UFC (670/705 (95\%)) and LSaC (183/205 (89\%)); $P=0.027$ and $P=0.011$ respectively (Fig. 3A).

In ADR-CS, LSeC (183/191 (96\%)) supported the diagnosis more frequently than UFC (187/217 (86\%)); $P<0.001$. Overnight $1 \mathrm{mg}$ DST (200/203 (99\%)) supported the diagnosis more frequently than both UFC $(187 / 217$ (86\%)) and LSaC (68/77 (88\%)); $P<0.01$ for both comparisons (Fig. 3B).
The diagnostic performance of the tests used to differentiate the etiologies of CS is shown in Table 3.

Measurement of cortisol post-HDDST was used in $30 \%$ of patients overall, $79 \%$ of whom were PIT-CS and 7\% ECT-CS. Assessment of peak post-CRH cortisol was used in $28 \%$ of patients, of whom $83 \%$ were PIT-CS and $5 \%$ ECT-CS. In those patients with negative pituitary MRI, HDDST supported the diagnosis of PIT-CS and ECT-CS in 90 and $88 \%$ of cases respectively $(P=0.54)$. The CRH test supported the diagnosis of PIT-CS and ECT-CS in 89 and $85 \%$ of cases $(P=0.57)$ (Table 4$)$.

Of patients with negative IPSS, HDDST supported the diagnosis of PIT-CS and ECT-CS in 100 and $82 \%$ respectively $(P=0.15)$. The CRH test was diagnostic in the only PIT-CS patient with negative IPSS (100\%) and in 75\% of ECT-CS $(P=0.43)$ (Table 4$)$.

\section{Pituitary imaging for the differential diagnosis of CS}

Pituitary MRI was performed in 928 patients (69\%), of whom 823 (89\%) had PIT-CS, 41 (4\%) had ADR-CS, 54 (6\%) had ECT-CS and 27 (1\%) had OTH-CS. As expected, PIT-CS patients (823/904 (91\%)) underwent a pituitary MRI more frequently than the other groups $(41 / 320$ (13\%) for ADR-CS, 54/80 (68\%) for ECT-CS and 10/37 $(27 \%)$ for OTH-CS; $(P<0.001))$. Pituitary MRI identified an adenoma in 628 of 809 (78\%) patients with PIT-CS having results available (440 microadenomas, 94 intrasellar macroadenomas and 94 macroadenomas with extrasellar extension) (Fig. 1). However an image indicating or suggesting a pituitary lesion compatible with a microadenoma was also documented in 10 of 37 (27\%) ADR-CS patients, 6 of 53 (11\%) ECT-CS patients and 4 of 9 (44\%) OTH-CS (Table 3). An intrasellar macroadenoma was observed in one ADR-CS patient (3\%). Of the 259 patients with a negative pituitary MRI, 181 (70\%) were subsequently diagnosed as having a PIT-CS, 26 ADR-CS (10\%), 47 ECT-CS (18\%) and 5 OTH-CS (2\%).

IPSS was performed in 310 patients overall, of whom $264(85 \%)$ were in the PIT-CS and 28 (9\%) in the ECT-CS groups. IPSS confirmed a pituitary source in 254 PIT-CS patients (96\%), whereas it did not support a pituitary origin of ACTH hypersecretion in 26 ECT-CS patients (93\%) (Table 3).

Of the 181 PIT-CS patients with a negative MRI, 129 (71\%) underwent IPSS; in 123 of them (95\%), the results 

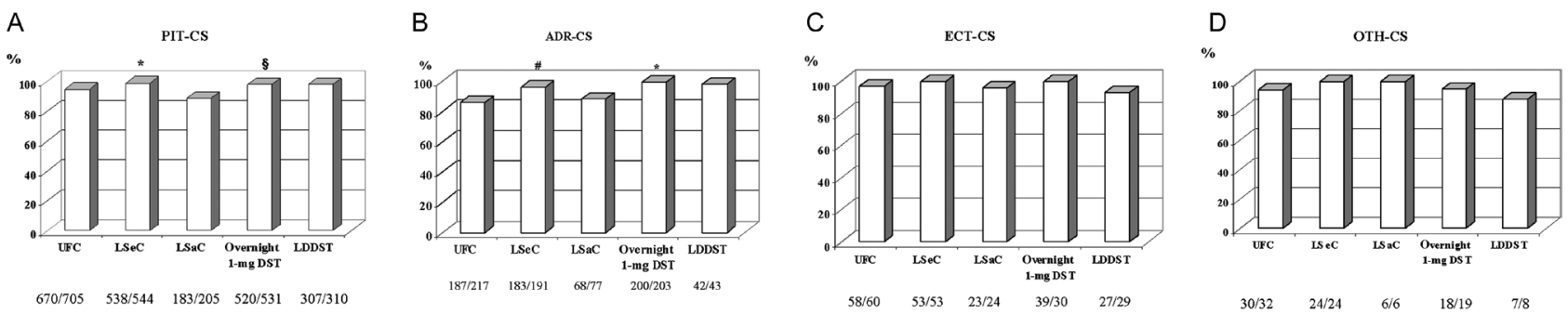

\section{Figure 3}

Comparison of tests supporting the diagnosis of CS within each etiologic group. (A) $* P<0.01$ vs UFC and LSaC; ${ }^{\S} P<0.05$ vs UFC and LSaC; (B) $* P<0.01$ vs UFC and LSaC; ${ }^{*} P<0.001$ vs UFC; (C) and (D) $P=$ NS. PIT-CS, pituitary-dependent CS; ADR-CS, adrenaldependent CS; ECT-CS, CS from an ectopic source; OTH-CS, CS from other etiologies; UFC, urinary free cortisol, LSeC, late-night serum cortisol; DST, dexamethasone suppression test and LSaC, late-night salivary cortisol.

were consistent with a pituitary source of ACTH secretion (Table 5). Inversely, IPSS indicated an extrapituitary source of ACTH in 22 of 23 (96\%) ECT-CS patients with a negative pituitary MRI.

Twenty-one PIT-CS patients also underwent pituitary CT, which was negative in 7 (33\%) of them. In three of these $7(43 \%)$ patients with negative CT, concomitant MRI documented a microdenoma.

\section{Adrenal imaging for the differential diagnosis of CS}

Normal adrenal glands were more frequently observed in PIT-CS $(171 / 267(64 \%))$ as compared with ADR-CS (107/298 (36\%)); $P<0.01$. A single adenoma (uni- or bilateral) was more frequently reported in ADR-CS $(196 / 298$ (66\%)) than in PIT-CS $(19 / 267(87 \%)) ; P<0.001$ (Table 3). Adrenal hyperplasia (uni- or bilateral) was more

Table 3 Performance of tests used for the differential diagnosis of CS. Numbers refer to tests with available results. Percentages are expressed in parentheses.

\begin{tabular}{|c|c|c|c|}
\hline & PIT-CS & ADR-CS & ECT-CS \\
\hline \multicolumn{4}{|c|}{ Differential diagnosis of PIT-CS vs ADR-CS } \\
\hline ACTH & 640/664 (96) & 223/225 (99) & - \\
\hline Positive pituitary MRI & $628 / 809(78)^{\#}$ & $10 / 37(27)$ & - \\
\hline \multicolumn{4}{|l|}{ Positive adrenal imaging* } \\
\hline Hyperplasia & 47/267 (18) & $41 / 298(14)$ & - \\
\hline Single adenoma & $19 / 267(7)$ & $196 / 298(66)^{\S}$ & - \\
\hline Multiple adenomas & $6 / 267(2)$ & $22 / 298(7)$ & - \\
\hline \multicolumn{4}{|c|}{ Differential diagnosis of PIT-CS vs ECT-CS } \\
\hline Positive pituitary MRI & $628 / 809(78) * *$ & - & $6 / 53(11)$ \\
\hline HDDST cortisol & $273 / 302(90)$ & - & $27 / 29(93)$ \\
\hline HDDST ACTH & $110 / 120(92)$ & - & $14 / 15(93)$ \\
\hline HDDST UFC & $136 / 145(94)$ & - & $14 / 16(93)$ \\
\hline CRH test cortisol (peak) & 269/309 (85) & - & $16 / 19(84)$ \\
\hline CRH test ACTH (peak) & 299/332 (90) & - & $16 / 19(84)$ \\
\hline Positive IPSS & $254 / 264(96)^{* *}$ & - & $2 / 28(7)$ \\
\hline \multicolumn{4}{|c|}{ Differential diagnosis of ADR-CS vs ECT-CS } \\
\hline ACTH & - & $223 / 225(99)$ & $54 / 58(93)$ \\
\hline Positive adrenal imaging* & - & & \\
\hline Hyperplasia & - & $41 / 298(14)$ & $20 / 34(59)^{\#}$ \\
\hline Single adenoma & - & $196 / 298(66) * *$ & $3 / 34(9)$ \\
\hline Multiple adenomas & - & $22 / 298(7)$ & $2 / 34(6)$ \\
\hline
\end{tabular}


Table 4 Percent of high-dose dexamethasone suppression test (HDDST) or corticotropin-releasing hormone test (CRH) supporting the diagnosis of either PIT-CS or ECT-CS. The data are classified based on findings on either pituitary magnetic resonance (MRI) or bilateral inferior petrosal sinus sampling (IPSS).

\begin{tabular}{|c|c|c|}
\hline Etiologic group & $\begin{array}{l}\text { Cortisol post-HDDST } \\
\text { supporting the } \\
\text { diagnosis }\end{array}$ & $\begin{array}{l}\text { Cortisol post-CRH } \\
\text { supporting the } \\
\text { diagnosis }\end{array}$ \\
\hline \multicolumn{3}{|l|}{ Overall } \\
\hline PIT-CS (\%) & 90 & 87 \\
\hline ECT-CS $(\%)$ & 93 & 84 \\
\hline$P$ value & 0.40 & 0.12 \\
\hline \multicolumn{3}{|c|}{ Patients with negative pituitary MRI } \\
\hline PIT-CS (\%) & 90 & 89 \\
\hline ECT-CS $(\%)$ & 88 & 85 \\
\hline$P$ value & 0.54 & 0.57 \\
\hline \multicolumn{3}{|c|}{ Patients with positive pituitary MRI } \\
\hline PIT-CS (\%) & 89 & 88 \\
\hline ECT-CS $(\%)$ & 100 & 0 \\
\hline$P$ value & 0.27 & 0.17 \\
\hline \multicolumn{3}{|c|}{ Patients with IPSS not supporting the pituitary origin } \\
\hline PIT-CS (\%) & 100 & 100 \\
\hline ECT-CS $(\%)$ & 82 & 75 \\
\hline$P$ value & 0.15 & 0.43 \\
\hline \multicolumn{3}{|c|}{ Patients with IPSS supporting the pituitary origin } \\
\hline PIT-CS (\%) & 87 & 78 \\
\hline ECT-CS $(\%)$ & 100 & - \\
\hline$P$ value & 0.40 & - \\
\hline
\end{tabular}

$P$ value refers to the comparison between PIT-CS and ECT-CS. ECT-CS, Cushing's syndrome from an ectopic source; IPSS, inferior petrosal sinus sampling; $\mathrm{MRI}$, magnetic resonance imaging; PIT-CS, pituitary Cushing's syndrome.

frequently observed in ECT-CS (20/34 (59\%)) than in ADR-CS (41/298 (14\%)); $P<0.01$ (Table 3).

\section{Discussion}

These ERCUSYN data show that the use of diagnostic tests for CS varies across European countries and partly differs from the currently available guidelines. Furthermore, late-night salivary cortisol (LSaC) assay is not frequently performed in the ERCUSYN participating centers despite being advocated as an easy and reliable tool to diagnose CS (24).

The most used measurement in the diagnostic work-up of CS is urinary free cortisol (UFC), which has been suggested as a first-line screening test in the current Endocrine Society guidelines (24). The other two recommended tests, the overnight $1 \mathrm{mg}$ DST and LSaC, were performed in 60 and $25 \%$ of ERCUSYN patients respectively. However the use of LSaC has increased over time among the ERCUSYN centers, indicating that this
Table 5 Number of PIT-CS patients having negative MRI who underwent inferior petrosal sinus sampling (IPSS) in each country and overall. Only tests with available results are reported. Data are presented as $n(\%)$.

\begin{tabular}{|c|c|c|c|}
\hline & $\begin{array}{l}\text { PIT-CS pts. with } \\
\text { negative MRI }\end{array}$ & IPSS performed & Positive IPSS \\
\hline \multicolumn{4}{|l|}{ Countries } \\
\hline Belgium & $3(10)$ & $3(100)$ & $3(100)$ \\
\hline Bulgaria & $9(24)$ & 0 & \\
\hline Croatia & $6(30)$ & $6(100)$ & $5(83)$ \\
\hline Czech & $4(17)$ & $2(50)$ & $2(100)$ \\
\hline France & $53(28)$ & 47 (89) & $44(94)$ \\
\hline Germany & $20(23)$ & $11(55)$ & $10(91)$ \\
\hline Greece & $9(23)$ & $5(56)$ & $5(100)$ \\
\hline Israel & $1(33)$ & $1(100)$ & $1(100)$ \\
\hline Italy & $1(6)$ & $1(100)$ & $1(100)$ \\
\hline Latvia & $1(25)$ & 0 & 0 \\
\hline Poland & $4(27)$ & 0 & 0 \\
\hline Romania & $1(13)$ & 0 & 0 \\
\hline Russia & $1(4)$ & $1(100)$ & 0 \\
\hline Slovenia & $8(31)$ & $6(75)$ & $6(100)$ \\
\hline Spain & $13(21)$ & $10(77)$ & $10(100)$ \\
\hline Sweden & $1(20)$ & $1(100)$ & $1(100)$ \\
\hline Switzerland & $3(75)$ & $3(100)$ & $3(100)$ \\
\hline Netherlands & 34 (19) & $24(71)$ & $24(100)$ \\
\hline Turkey & $1(13)$ & $1(100)$ & $1(100)$ \\
\hline UK & $8(26)$ & $7(88)$ & 7 (100) \\
\hline Total & $181(22)$ & $129(71)$ & $123(95)$ \\
\hline
\end{tabular}

measurement is progressively being recognized as a useful diagnostic tool in suspected CS across Europe. LSaC has convincingly been proposed as a first-line diagnostic test in CS in 1998, and Endocrine Society guidelines endorsed its use in $2008(24,26)$. Our data reflect that a lapse of time is needed to introduce evidence-based recommendations into the daily clinical practice. In addition, it should be borne in mind that one-quarter of patients were diagnosed as having CS before 2008, when the Endocrine Society guidelines were published.

Recent studies showed that $\mathrm{LSaC}$ is a noninvasive, valid alternative to UFC for diagnosing CS in light of its simple collection procedure and elevated diagnostic performance $(10,27)$. In fact, $\mathrm{LSaC}$ accurately reflects the plasma free cortisol concentrations irrespective of the saliva production rate and corticosteroid-binding globulin (CBG) variability (12).

Loss of nighttime cortisol nadir in saliva is a hallmark of endogenous hypercortisolism, and reliably allows the identification of mild CS in those patients having normal or slightly elevated UFC $(9,16,23,27,28)$. LSaC supported the diagnosis of CS in $88 \%$ of ERCUSYN patients with this measurement available, which was similar to the 93\% observed for UFC (data not shown). Likewise, no 
differences were reported in the diagnostic performance of LSaC and UFC within each etiologic group. Despite its good performance and practical advantages, measurement of $\mathrm{LSaC}$ is not as common as expected across Europe, likely due to misconcepted cost-effectiveness concerns and still limited access to the assay in some institutions (29). There is also some evidence that its performance is variable across centers (30). However several relatively inexpensive immunoassays are now commercially available, and have been proven to yield a good diagnostic performance (12, 31 ). On the other hand, the cost of liquid chromatography/ tandem mass spectrometry (LC/TMS), which avoids the cross-reactivity between cortisol and synthetic steroids, is rapidly decreasing, making it a feasible option even for routine diagnostic laboratories (32). European authorities should make an effort to set up a program for quality assessment and harmonization of different LSaC assays, or promote the diffusion of a well-validated method, which can be easily adopted by all the reference laboratories (12, 32 ). Of note, groups entering more than 20 patients into ERCUSYN were more likely to include LSaC (or LSeC) assessment in the work-up of CS as compared with those entering less than 20 patients, suggesting that the clinical experience of a given center and/or the availability of adequate assays are the important factors impacting on the diagnostic choices.

Noteworthily, MSeC and ACTH were the most frequently reported tests in the ERCUSYN diagnosis section. MSeC is not advisable in the diagnostic work-up of CS due to its low reliability (33). Accordingly, MSeC supported the diagnosis in only half of $82 \%$ of ERCUSYN patients in whom it was performed (data not shown). It should be emphasized that a significant number of MSeC assays reported in the ERCUSYN were likely to be performed not as independent tests, but within different dynamic tests, such as diurnal cortisol rhythm assessment, LDDST and HDDST. Because the ERCUSYN database is not able to clarify in what context the MSeC measurement was performed, data on this test should be interpreted with caution.

Measurement of ACTH is useful in the differential diagnosis of CS (1). A single reported ACTH measurement in the ERCUSYN supported the diagnosis of PIT-CS and ECT-CS in 96 and 93\% of patients respectively, which was similar to the 99\% reported in ADR-CS. However recent evidence suggests that ACTH assessment may be misleading in CS, mainly depending on the assay used, in that up to $40 \%$ of patients with ACTHindependent CS might have normal corticotrophin values (34). Moreover, ACTH should be measured at least from two independent samples in order to avoid misinterpretation in ACTH-dependent CS, due to its episodic secretion (1) and because it requires stringent pre-analytic conditioning. We cannot exclude that some ERCUSYN centers chose to uniquely report a single ACTH value supporting the diagnosis among multiple samplings. Another limitation of the ERCUSYN is that it does not allow differentiating those tests, which have been performed in a given center to screen patients suspected of having hypercortisolism, from those used to confirm the diagnosis of CS. However most of the ERCUSYN participating centers are tertiary hospitals in their countries and, therefore they are more likely to admit patients referred from peripheral institutions to obtain the diagnostic confirmation of CS.

Despite these limitations, our data clearly showed a wide inter-country heterogeneity in local testing protocols for CS. When considering each of the most frequently performed tests, the use ranged broadly from around 10\% to almost $100 \%$, across the countries. This reflects the lack of a common European diagnostic strategy for this rare condition, which may partly be accounted for by logistic, economic, political and cultural differences. It would seem pertinent to attempt a more uniform approach throughout Europe for the patient with CS, aimed at maximizing the patient's benefit on the one side, and considering cost rationalization on the other.

It should be acknowledged that missing or incomplete information is a potential shortcoming of a multicenter registry like the ERCUSYN, but the careful review for data quality control, which has been performed prior to data analysis, should have, in our opinion, attenuated the impact of this limitation on data reliability.

LSeC and $1 \mathrm{mg}$ overnight DST were proven to support the diagnosis of both PIT-CS and ADR-CS more frequently than UFC, in line with previous reports. Invitti et al found that UFC levels fell within the normal range in $9 \%$ of PIT-CS and 15\% of ADR-CS patients (33). It is well known that several technical issues might limit the reliability of the most common UFC immunoassays, including interference by metabolites and conjugates, inter-assay variability and intra-subject day-to-day variations (29). Of note, Rossi et al. did not report any difference in the UFC levels between patients with an adrenal mass and concomitant 'subclinical' hypercortisolism as compared with healthy controls (35). Although the diagnosis of a nonfunctioning adrenal incidentaloma was an exclusion criterion of ERCUSYN, we cannot rule out that some patients with subtle hypercortisolism associated with an adrenal incidentaloma were also included. 
The usefulness of HDDST and CRH test for the differential diagnosis of ACTH-dependent CS is highly questionable due to their low accuracy (24). Accordingly, ERCUSYN data have shown that HDDST and CRH did not identify ECT-CS in 18 and 25\% of patients respectively, who were subsequently found to have ectopic ACTH hypersecretion, supporting the recommendation that these tests should be performed only when IPSS is not feasible or available (1). Notably, a significant decrease in the use of CRH test (but not HDDST) has been observed over time in the ERCUSYN centers. This is likely due to the poor performance of this test and the limited availability of $\mathrm{CRH}$ in different countries, along with the continuous improvement of the imaging techniques, which more effectively support the differential diagnosis of hypercortisolism as compared with 'traditional' testing. This is also expected to significantly reduce the use of HDDST in the future.

It would seem, therefore, that while some of the tests recommended by the guidelines should be performed more frequently than has been done in recent years, others which still seem to be relied upon, should probably be avoided, given their limited practical utility.

Although not specifically mentioned in the current Endocrine Society guidelines, it is widely accepted that MRI is the first-choice imaging modality during the diagnostic process of ACTH-dependent hypercortisolemic states. The ERCUSYN data are compatible with these suggestions, since $89 \%$ of PIT-CS patients underwent pituitary MRI. In line with the previous reports, the diagnostic accuracy of MRI in the differential diagnosis of ACTH-dependent CS was not optimal, showing a sensitivity of $78 \%$ and a specificity of $89 \%(36,37)$. In addition to this, a pituitary incidentaloma was found in $2 \%$ of patients who underwent an MRI, in whom an ACTH-secreting adenoma was subsequently ruled out as the cause of their hypercortisolism $(38,39)$. It should be emphasized that, while these ERCUSYN data refer to MRI equipment in use over the last 15 years, the rapid development of more sensitive devices might provide different results in the near future (40).

Of note, $22 \%$ of the PIT-CS patients had a normal pituitary MRI and, among those, two-thirds underwent IPSS, which confirmed a pituitary source of hypercortisolism in 95\%. Overall, sensitivity and specificity of IPSS were 96 and 93\% respectively, in accordance with the previous studies, which described a sensitivity ranging from 81 to $100 \%$ and a specificity from 90 to $95 \%$ (40). Thus, this invasive procedure appears to yield a better diagnostic accuracy than MRI and dynamic testing used for the differential diagnosis of ACTH-dependent CS. Therefore it should be considered a useful diagnostic tool to confirm a pituitary source of ACTH overproduction, mainly in those patients with sustained hypercortisolism having a negative MRI and/or inconclusive biochemical evaluation (36, 37, 39, $40,41,42,43)$. Although the ERCUSYN results, obtained in the largest series published to date, suggest that IPSS is extensively used across Europe, it was not reported in some countries, indicating that its use should be further implemented, for instance, by supporting technical skills training and procedure supplies acquisition.

In conclusion, our data demonstrate that there is heterogeneity throughout Europe in the biochemical testing performed to detect hypercortisolism and diagnose CS. An effort should be taken in order to promote tests with better accuracy while limiting the use of those measurements, which are not recommended in the current guidelines. Notably, a change over time in the use of some tests has been observed. These results emphasize the importance of elaborating a common European strategy for the diagnosis and follow-up of CS, identifying the most accurate and cost-effective diagnostic approach to this rare disease, while taking into account specific inter-country differences.

\section{Supplementary data}

This is linked to the online version of the paper at http://dx.doi.org/10.1530/ EJE-16-0967.

\section{Declaration of interest}

$S \mathrm{M}$ W received financial support, research grants and consultant or speaker fees from Ipsen, Novartis, Pfizer, HRA and Strongbridge. A T received financial support, research grants and consultant fees from Novartis and HRA Pharma. C J S has received lecture fees, consultancy remuneration or research support from HRA Pharma, Novartis and Strongbridge. T B received financial support, research grants and consultant or speaker fees from Ipsen, Novartis, Pfizer, Sandoz and Strongbridge. The authors declare that there is no conflict of interest that could be perceived as prejudicing the impartiality of the research reported.

\section{Funding}

ERCUSYN was set up with funding from the EU (PHP 800200) and been supported by unrestricted grants from Novartis, Ipsen, HRA and the European Society of Endocrinology.

\section{Acknowledgements}

ERCUSYN Study Group: A Ambrogio, Istituto Auxologico Italiano IRCCS, University of Milan, Italy; G Aranda, Department of Endocrinology, Hospital Clinic Barcelona, IDIBAPS, UB, Barcelona, Spain; M Arosio, Unit of Endocrine Diseases \& Diabetology, Department of Clinical Sciences and Community Health, University of Milan, Milan, Italy; M Balomenaki, 
Athens Polyclinic General Hospital, Evangelismos Hospital, Athens, Greece; P Beck-Peccoz, Endocrinology and Diabetology Unit, Fondazione IRCCS Ca' Granda - Ospedale Maggiore Policlinico, University of Milan, Milan, Italy; C Berr-Kirmair, Medizinische Klinik und Poliklinik IV, Campus Innestadt, Klinikum der Universität München, München, Germany; J Bollerslev, Section of Specialized Endocrinology, Oslo University Hospital, and Faculty of Medicine, University in Oslo, Oslo, Norway; D Carvalho, Hospital de San Joao, Porto, Portugal; F Cavagnini, Istituto Auxologico Italiano IRCCS, Milan, Italy; E Christ, University Hospital of Bern, Inelspital, Division of Endocrinology, Diabetology and Clinical Nutrition, Bern, Switzerland; F Demtröder Zentrum fur Endokrinologie, Diabetologie, Rheumatologie Dr Demtröder \& Kollegen im MVZ Dr Eberhard \& Partner und Klinikum Dortmund, Germany; J Denes, Division of Endocrinology, 2nd Department of Medicine, State Health Center, Budapest, Hungary; C Dimopoulou, Max-Planck-Gessellschaft zur Forderung der Wissenschaften e.V., Munich, Germany; A Dreval, Moscow Regional Research Clinical Institute n.a. Vladimirsky, Moscow, Russia; T Dusek, Department of Endocrinology, University Hospital Zagreb, School of Medicine University of Zagreb, Kispaticeva 12, 10000 Zagreb, Croatia; E Erdinc, Uludag University School of Medicine, Bursa, Turkey; J A Evang, Section of Specialized Endocrinology, Oslo University Hospital, and Faculty of Medicine, Oslo University in Oslo, Oslo, Norway; J Fazel, Medizinische Klinik und Poliklinik IV, Campus Innestadt, Klinikum der Universität München, München, Germany; S Fica, Elias Hospital, Bucharest, Romania; E Ghigo, Molinette Hospital, Department of Internal Medicine, Turin, Italy; M Goth, Division of Endocrinology, 2nd Department of Medicine, State Health Center, Budapest, Hungary; Y Greenman, Institute of Endocrinology, Metabolism and hypertension, Tel Aviv, Israel; V Greisa, Medizinische Universitat Wien, Wien, Austria; I Halperin, Department of Endocrinology, Hospital Clinic Barcelona, IDIBAPS, UB, Barcelona, Spain; FA Hanzu, Department of Endocrinology, Hospital Clinic Barcelona, IDIBAPS, UB, Barcelona, Spain; A Hermus, Radboud University Medical Center, Nijmegen The Netherlands; G Johannsson, Goteborg University, Goteborg, Sweden; P Kamenicky, Univ Paris-Sud, Université Paris-Saclay UMR-S1185, Le Kremlin Bicêtre, Paris F-94276, France, Assistance Publique-Hôpitaux de Paris, Hôpital de Bicêtre, Service de Endocrinologie et des Maladies de la Reproduction, Le Kremlin Bicêtre, Paris, F-94275, France, Institut National de la Santé et de la Recherche Médicale U1185, Le Kremlin Bicêtre, Paris F-94276, France; A Kasperlik-Zaluska, Centre for Postgraduate Medical Education, Warsaw, Poland; J Kirchner, Division of Clinical Endocrinology, Department of Medicine CCM, Charité- Universitätsmedizin, Berlin, Germany; I Kraljevic, Department of Endocrinology, University Hospital Zagreb, Kispaticeva 12, 10000 Zagreb, Croatia; A Kruszynska, Centre for Postgraduate Medical Education, Warsaw, Poland; I Lambrescu, Elias Hospital, Bucharest, Romania; S Lang, Max-Planck-Gessellschaft zur Forderung der Wissenschaften e.V., Munich, Germany; A Luger, Medizinische Universitat Wien, Wien, Austria; N Marpole, Christie Hospital, NHS Trust, Manchester, UK; S Martin, Elias Hospital, Bucharest, Romania; M Martinie, Service d'EndocrinologieDiabétologie-Nutrition, Grenoble Cedex, France; O Moros, Zentrum fur Endokrinologie, Diabetologie, Rheumatologie Dr Demtröder \& Kollegen im MVZ Dr Eberhard \& Partner und Klinikum Dortmund; J Newell-Price, The University of Sheffield, Sheffield, UK; M Orbetzova, Clinic of Endocrinology and Metabolic Diseases, 'Sv.Georgy' University Hospital, Medical University, Plovdiv, Bulgaria; I Paiva, Hospitais da Universidade de Coimbra; F Pecori Giraldi, Istituto Auxologico Italiano IRCCS, University of Milan, Italy; AM Pereira, Leiden University Medical Center, Leiden, The Netherlands; J Pickel, Max-Planck-Gessellschaft zur Forderung der Wissenschaften e.V., Munich, Germany; V Pirags, Pauls Stradinš Clinical University Hospital, University of Latvia, Riga, Latvia; O Ragnarsson, Goteborg University, Goteborg, Sweden; AD Reghina, Elias Hospital, Bucharest, Romania; P Riesgo, Neurosurgery Department, Hospital Universitario de la Ribera, Alzira, Spain; M Roberts, Christie Hospital, NHS Trust, Manchester, UK; S Roerink, Radboud University Medical Center, Nijmegen, The Netherlands; O Roig, IIB-Sant Pau and Department of Endocrinology/Medicine, Hospital Sant Pau, UAB, and Centro de Investigación Biomédica en Red de Enfermedades Raras (CIBER-ER, Unidad 747), ISCIII; C Rowan, Christie Hospital, NHS Trust,
Manchester, UK; P Rudenko, Estonian Endocrine Society, Tallinn, Estonia; M A Sahnoun, Aix-Marseille Université, CNRS, CRN2M UMR 7286, 13344 Cedex 15, Marseille, and APHM, Hôpital Conception, Marseille, France; J Salvador, University of Navarra, Pamplona, Spain; HA Sigurjonsdottir, Landspitali University Hospital, Reykjavik, Iceland and Faculty of Medicine, University of Iceland, Reykjavik, Iceland; T Skoric Polovina, Department of Endocrinology, University Hospital Zagreb, Kispaticeva 12, 10000 Zagreb, Croatia; R Smith, Oxford Radcliffe Hospitals NHS Trust, Oxford, UK; B Stachowska, Department of Endocrinology, Diabetology and Isotope Therapy Wroclaw Medical University, Wroclaw, Poland; G Stalla, Max-Planck-Gessellschaft zur Forderung der Wissenschaften e.V., Munich, Germany; J Tôke, 2nd Department of Medicine, Semmelweis University, Budapest, Hungary; E Ubina, Division of Endocrinology, 2nd Department of Medicine, State Health Center, Budapest, Hungary; S Vinay, Christie Hospital, NHS Trust, Manchester, UK; M Wagenmakers, Radboud University Medical Center, Nijmegen, The Netherlands; S Werner, Praxis für Endokrinologie Droste, Oldenburg, Germany; J Young, Univ ParisSud, Université Paris-Saclay UMR-S1185, Le Kremlin Bicêtre, Paris F-94276, France, Assistance Publique-Hôpitaux de Paris, Hôpital de Bicêtre, Service de Endocrinologie et des Maladies de la Reproduction, Le Kremlin Bicêtre, Paris, F-94275, France, Institut National de la Santé et de la Recherche Médicale U1185, Le Kremlin Bicêtre, Paris F-94276, France; P Zdunowski, Centre for Postgraduate Medical Education, Warsaw, Poland; K Zopf Division of Clinical Endocrinology, Department of Medicine CCM, CharitéUniversitätsmedizin, Berlin, Germany; S Zopp, Medizinische Klinik und Poliklinik IV, Campus Innestadt, Klinikum der Universität München, München, Germany; I Zosin, Romanian Society for Endocrinology, Timisoara, Romania.

\section{References}

1 Newell-Price J, Trainer P, Besser M \& Grossman A. The diagnosis and differential diagnosis of Cushing's syndrome and pseudo-Cushing's states. Endocrine Reviews 199819 647-672. (doi:10.1210/er.19.5.647)

2 Cronin C, Igoe D, Duffy MJ, Cunningham SK \& McKenna TJ. The overnight dexamethsone test is a worthwhile screening procedure. Clinical Endocrinology 199033 27-33. (doi:10.1111/j.1365-2265.1990. tb00462.x)

3 Papanicolau DA, Yanovski JA, Cutler GB Jr, Chrousos GP \& Nieman LK. A single midnight serum cortisol measurement distinguishes Cushing's syndrome from pseudo-Cushing's states. Journal of Clinical Endocrinology and Metabolism 199883 1163-1167. (doi:10.1210/jc.83.4.1163)

4 Castro M, Elias PC, Quidute AR, Halah FP \& Moreira AC. Out-patient screening for Cushing's syndrome: the sensitivity of the combination of circadian rhythm and overnight dexamethasone suppression salivary cortisol tests. Journal of Clinical Endocrinology and Metabolism 199984 878-882. (doi:10.1210/jc.84.3.878)

5 Gorges R, Knappe G, Gerl H, Ventz M \& Stahl F. Diagnosis of Cushing's syndrome: re-evaluation of midnight plasma cortisol vs. urinary free cortisol and low-dose dexamethasone suppression test in a large patient group. Journal of Endocrinological Investigation 199922 241-249. (doi:10.1007/BF03343551)

6 Moro M, Putignano P, Losa M, Invitti C, Maraschini C \& Cavagnini F. The desmopressin test in the differential diagnosis between Cushing's disease and pseudo-Cushing states. Journal of Clinical Endocrinology and Metabolism 200085 3569-3574. (doi:10.1210/jcem.85.10.6862)

7 Findling JW, Raff H \& Aron DC. The low-dose dexamethasone suppression test: a reevaluation in patients with Cushing's syndrome. Journal of Clinical Endocrinology and Metabolism 200489 1222-1226. (doi:10.1210/jc.2003-030207)

8 Yaneva M, Mosnier-Pudar H, Dugue MA, Grabar S, Fulla Y \& Bertagna X. Midnight salivary cortisol for the initial diagnosis of Cushing's syndrome of various causes. Journal of Clinical Endocrinology and Metabolism 200489 3345-3351. (doi:10.1210/jc.2003-031790) 
9 Reimondo G, Allasino B, Bovio S, Paccotti P, Angeli A \& Terzolo M. Evaluation of the effectiveness of midnight serum cortisol in the diagnostic procedures for Cushing's syndrome. European Journal of Endocrinology 2005153 803-809. (doi:10.1530/eje.1.02042)

10 Viardot A, Huber P, Puder JJ, Zulewski H, Keller U \& Muller B. Reproducibility of nighttime salivary cortisol and its use in the diagnosis of hypercortisolism compared with urinary free cortisol and overnight dexamethasone suppression test. Journal of Clinical Endocrinology and Metabolism 200590 5730-5736. (doi:10.1210/ jc.2004-2264)

11 Pecori-Giraldi F, Ambrogio G, De Martin M, Fatti LM, Scacchi M \& Cavagnini F. Specificity of first-line tests for the diagnosis of Cushing's syndrome: assessment in a large series. Journal of Clinical Endocrinology and Metabolism 200792 4123-4109. (doi:10.1210/jc.2007-0596)

12 Raff H. Utility of salivary cortisol measurements in Cushing's syndrome and adrenal insufficiency. Journal of Clinical Endocrinology and Metabolism 200994 3647-3655. (doi:10.1210/jc.2009-1166)

13 Workman RJ, Vaughn WK \& Stone WJ. Dexamethasone suppression testing in chronic renal failure: pharmacokinetics of dexamethasone and demonstration of a normal hypothalamic-pituitary-adrenal axis. Journal of Clinical Endocrinology and Metabolism 198663 741-746. (doi:10.1210/jcem-63-3-741)

14 Lin CL, Wu TJ, Machacek DA, Jiang NS \& Kao PC. Urinary free cortisol and cortisone determined by high performance liquid chromatography in the diagnosis of Cushing's syndrome. Journal of Clinical Endocrinology and Metabolism 199782 151-155. (doi:10.1210/ jcem.82.1.3687)

15 Ness-Abramof R, Nabriski D, Apovian CM, Niven M, Weiss E, Shapiro MS \& Shenkman L. Overnight dexamethasone suppression test: a reliable screen for Cushing's syndrome in the obese. Obesity Research 200210 1217-1221. (doi:10.1038/oby.2002.166)

16 Putignano P, Bertolini M, Losa M \& Cavagnini F. Screening for Cushing's syndrome in obese women with and without polycystic ovary syndrome. Journal of Endocrinological Investigation $2003 \mathbf{2 6}$ 539-544. (doi:10.1007/BF03345217)

17 Klose M, Lange M, Rasmussen AK, Skakkebaek NE, Hilsted L, Haug E, Anderes M \& Feldt-Rasmussen U. Factors influencing the adrenocorticotropin test: role of contemporary cortisol assays, body composition, and oral contraceptive agents. Journal of Clinical Endocrinology and Metabolism 200792 1326-1333. (doi:10.1210/ jc.2006-1791)

18 Valassi E, Swearingen B, Lee H, Nachtigall LB, Donoho DA, Klibanski A \& Biller BMK. Concomitant medication use can confound interpretation of the combined dexamethasone-corticotropin releasing hormone test in Cushing's syndrome. Journal of Clinical Endocrinology and Metabolism 200994 4851-4859. (doi:10.1210/ jc.2009-1500)

19 Mullan K, Black N, Thiraviaraj A, Bell PM, Burgess C, Hunter SJ, McCance DR, Leslie H, Sheridan B \& Atkinson AB. Is there value in routine screening for Cushing's syndrome in patients with diabetes? Journal of Clinical Endocrinology and Metabolism 201095 2262-2265. (doi:10.1210/jc.2009-2453)

20 Friedman TC, Ghods DE, Shahinian HK, Zachery L, Shayesteh N, Seasholtz S, Zuckerbraun E, Lee ML \& McCutcheon IE. High prevalence of normal tests assessing hypercortisolism in subjects with mild and episodic Cushing's syndrome suggests that the paradigm for diagnosis and exclusion of Cushing's syndrome requires multiple testing. Hormone and Metabolic Research 201042 874-881. (doi:10.1055/s-0030-1263128)

21 Ceccato F, Antonelli G, Barbot M, Zilio M, Mazzai L, Gatti R, Zaninotto M, Mantero F, Boscaro M, Plebani M et al. The diagnostic performance of urinary free cortisol is better than the cortisol:cortisone ratio in detecting de novo Cushing's syndrome: the use of LC-MS/MS method in routine clinical practice. European Journal of Endocrinology 2014171 1-7. (doi:10.1530/EJE-14-0061)
22 Ritzel K, Beuschlein F, Berr C, Osswald A, Reisch N, Bidlingmaier M, Schneider H, Honegger J, Geyer LL, Schopohl J et al. ACTH after 15 min distinguishes between Cushing's disease and ectopic Cushing's syndrome: a proposal for a short and simple CRH test. European Journal of Endocrinology 2015173 197-204. (doi:10.1530/EJE-14-0912)

23 Nunes ML, Vattaut S, Corcuff JB, Rault A, Loiseau H, Gatta B, Valli N, Letenneur L \& Tabarin A. Late-night salivary cortisol for diagnosis of overt and subclinical Cushing's syndrome in hospitalized and ambulatory patients. Journal of Clinical Endocrinology and Metabolism 200994 456-462. (doi:10.1210/jc.2008-1542)

24 Nieman LK, Biller BMK, Findling JW, Newell-Price J, Savage MO, Stewart PM \& Montori VM. The diagnosis of Cushing's syndrome: an endocrine society clinical practice guideline. Journal of Clinical Endocrinology and Metabolism 200893 1526-1540. (doi:10.1210/ jc.2008-0125)

25 Valassi E, Santos A, Yaneva M, Tóth M, Strasburger CJ, Chanson P, Wass JA, Chabre O, Pfeifer M, Feelders RA et al. The European registry on Cushing's syndrome: 2 year experience. Baseline demographic and clinical characteristics. European Journal of Endocrinology 2011165 383-392. (doi:10.1530/EJE-11-0272)

26 Raff H, Raff JL \& Findling JW. Late-night salivary cortisol as a screening test for Cushing's syndrome. Journal of Clinical Endocrinology and Metabolism 199883 2681-2686. (doi:10.1210/jcem.83.8.4936)

27 Elias PCL, Martinez EZ, Barone BFC, Mermejo LM, Castro M \& Moreira AC. Late-night salivary cortisol has a better performance than urinary free cortisol in the diagnosis of Cushing's syndrome. Journal of Clinical Endocrinology and Metabolism 201499 2045-2051. (doi:10.1210/jc.2013-4262)

28 Kidambi S, Raff H \& Findling JW. Limitations of nocturnal salivary cortisol and urine free cortisol in the diagnosis of mild Cushing's syndrome. European Journal of Endocrinology 2007157 725-731. (doi:10.1530/EJE-07-0424)

29 Pecori-Giraldi F \& Ambrogio AG. Variability in laboratory parameters used for management of Cushing's syndrome. Endocrine $2015 \mathbf{5 0}$ 580-589. (doi:10.1007/s12020-015-0676-9)

30 Elamin MB, Murad Hassan M, Mullan R, Erickson D, Harris K, Nadeem S, Ennis R, Erwin PJ \& Montori VM. Accuracy of the diagnostic tests for Cushing's syndrome: a systematic review and metaanalysis. Journal of Clinical Endocrinology and Metabolism 200893 1553-1562. (doi:10.1210/jc.2008-0139)

31 Carroll T, Raff H \& Findling JW. Late-night salivary cortisol for the diagnosis of Cushing's syndrome: a meta-analysis. Endocrine Practices 200915 335-342. (doi:10.4158/EP09023OR)

32 Monaghan PJ, Keevil BG, Stewart P \& Trainer PJ. Case for the wider adoption of mass spectrometry-based adrenal steroid testing, and beyond. Journal of Clinical Endocrinology and Metabolism 201499 4434-4437. (doi:10.1210/jc.2014-2258)

33 Invitti C, Pecori Giraldi F, De Martin M \& Cavagnini F. Diagnosis and management of Cushing's syndrome: results of an Italian multicentre study. Journal of Clinical Endocrinology and Metabolism $1999 \mathbf{8 4}$ 440-448. (doi:10.1210/jcem.84.2.5465)

34 Pecori Giraldi F, Saccani A \& Cavagnini F. Assessment of ACTH assay variability; a multicenter study. European Journal of Endocrinology 2011 164 505-512. (doi:10.1530/EJE-10-0962)

35 Rossi R, Tauchmanova L, Luciano A, Di Martino M, Battista C, Del Viscovo L, Nuzzo V, Lombardi G. Subclinical Cushing's syndrome in patients with adrenal incidentaloma: clinical and biochemical features. Journal of Clinical Endocrinology and Metabolism 200085 1440-1448. (doi:10.1210/jcem.85.4.6515)

36 Tabarin A, Laurent F, Catargi B, Olivier-Puel F, Lescene R, Berge J, Galli FS, Drouillard J, Roger P \& Guerin J. Comparative evaluation of conventional and dynamic magnetic resonance imaging of the pituitary gland for the diagnosis of Cushing's disease. Clinical Endocrinology 199849 293-300. (doi:10.1046/j.13652265.1998.00541.x) 
37 Patronas N, Bulakbasi N, Startakis CA, Lafferty A, Oldfield EH, Doppman J \& Nieman LK. Spoiled gradient recalled acquisition in the steady state technique is superior to conventional post-contrast spin echo technique for magnetic resonance imaging detection of adrenocorticotropin-secreting pituitary tumors. Journal of Clinical Endocrinology and Metabolism 200388 1565-1569. (doi:10.1210/ jc.2002-021438)

38 Buurman H \& Saeger W. Subclinical adenomas in post-mortem pituitaries: classification and correlations to clinical data. European Journal of Endocrinology $2006 \mathbf{1 5 4}$ 753-758. (doi:10.1530/eje.1.02107)

39 Mehta GU, Montgomery BK, Raghavan P, Sharma S, Nieman LK, Patronas N, Oldfield EH \& Chittiboina P. Different imaging characteristics of concurrent pituitary adenomas in a patient with Cushing's disease. Journal of Clinical Neuroscience 201522 891-894. (doi:10.1016/j.jocn.2015.01.001)

40 Pecori Giraldi F, Cavallo LM, Tortora F, Pivonello R, Colao A, Cappabianca P \& Mantero F. The role of inferior petrosal sinus sampling in ACTH-dependent Cushing's syndrome: review and joint opinion statement by members of the Italian Society for Endocrinology, Italian Society for Neurosurgery, and Italian Society for Neuroradiology. Neurosurgical Focus 201538 E(5). (doi:10.3171/2014.11.focus14766)

41 Aron Dc, Raff H \& Findling JW. Effectiveness versus efficacy: the limited value in clinical practice of high dose dexamethasone suppression testing in the differential diagnosis of adrenocorticotropin-dependent Cushing's syndrome. Journal of Clinical Endocrinology and Metabolism 199782 1780-1785. (doi:10.1210/jcem.82.6.3991)

42 Reimondo G, Paccotti P, minetto M, Termine A, Stura G, Bergui M, Angeli A \& Terzolo $\mathrm{M}$. The corticotrophin-releasing hormone test is the most reliable noninvasive method to differentiate pituitary from ectopic ACTH secretion in Cushing's syndrome. Clinical Endocrinology 200358 718-724. (doi:10.1046/j.1365-2265.2003.01776.x)

43 Zampetti B, Grossrubatscher Dalino Ciaramella P, Boccardi E \& Loli P. Bilateral inferior petrosal sinus sampling. Endocrine Connections 2016 5 R12-R25. (doi:10.1530/EC-16-0029)

Received 24 November 2016

Revised version received 13 February 2017

Accepted 21 February 2017 